\title{
A novel quasi-peak-detector for time-domain EMI-measurements
}

\author{
F. Krug, S. Braun, and P. Russer \\ Institute for High-Frequency Engineering, Technische Universität München, Arcisstrasse 21, 80333 Munich, Germany
}

\begin{abstract}
In this paper, an advanced ultra-fast, broadband time domain EMI measurement system is described. Measurements were performed in the $30-1000 \mathrm{MHz}$ range. The digital signal processing of EMI measurements allows to emulate in real-time the modes of conventional analogous equipment, e.g. Peak-, Average-, RMS- and Quasi-PeakDetector. With the presented time domain measurement system the measurement time can be reduced by a factor of 10. A novel signal recording routine for time-domain EMI (TDEMI) measurements and Quasi-Peak-Detection is described. Measurement results obtained from the investigation of a drill machine, monitor and laptop obtained with the timedomain electromagnetic interference (TDEMI) measurement system are discussed. The results obtained with the described system have been compared with measurements performed with a conventional EMI receiver.
\end{abstract}

\section{Introduction}

Due to the rapid development of new electronic products and due to emerging new technologies the ability to achieve and to improve electromagnetic compatibility is a major challenge in development of electronic products. EMC and EMI measurement equipment which allows to extract comprehensive and accurate information within short measurement times will allow to reduce the costs and to improve the quality in circuit and system development. In the past and currently, radio noise and electromagnetic interference (EMI) are measured and characterized by superheterodyne radio receivers. The disadvantage of this method is the quite long measurement time of typically $30 \mathrm{~min}$ for a frequency band from $30 \mathrm{MHz}$ to $1 \mathrm{GHz}$. Since such a long measurement time results in high test costs, it is important to look up for possibilities to reduce the measurement time without loss of quality. The digital processing of EMI measurements using

Correspondence to: F. Krug

(fkrug@ieee.org)
Fourier Transform allows the decomposition of a signal measured in time-domain into its spectral components. In general the digital processing of EMI measurements allows to emulate in real-time the various modes of conventional analogous equipment and it is also possible to introduce new concepts of analysis (Krug, 2002a).

In the following a new signal recording routine for time-domain EMI (TDEMI) measurement and Quasi-PeakDetection is described. The measurement routine inhibits intelligent triggering to record the EMI signal. This makes it possible to reduce data enormously. A method to reconstruct an equivalent of the original signal is described. An accurate time-domain measurement with Average-, RMS- and QuasiPeak-Detection on all signal types are possible.

\section{Advanced TDEMI measurement concept}

A detailed hardware description of the used TDEMI measurement system has already been presented in Krug (2002b, 2003). In Fig. 1 the advanced TDEMI measurement concept is shown. The data acquisition process for the timedomain measurement starts with the sample process of the oscilloscope. Then the spectra via the Short-Time-FourierTransform (STFT) is digitally computed. After the STFT the errors due to the frequency characteristics of antenna, transmission line, amplifier and anti-aliasing filter are corrected by signal processing. After this the analysis of Peak-, RMS-, Average- and Quasi-Peak-values of the EMI signal is performed.

\section{Automated TDEMI measurement algorithm for class C signals}

When measuring signal with transient envelope, the pulses can record separately, which allows to sample pulses with different amplitudes with an optimum vertical resolution of the Analog-to-Digital-Converter (ADC). First the measurement routine with one vertical resolution is described. 


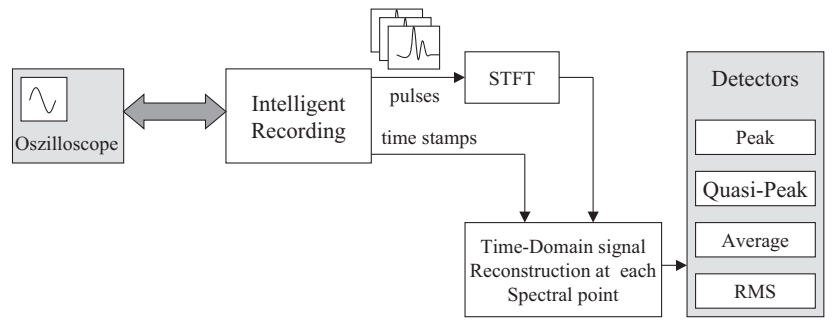

Fig. 1. Advanced TDEMI measurement concept.

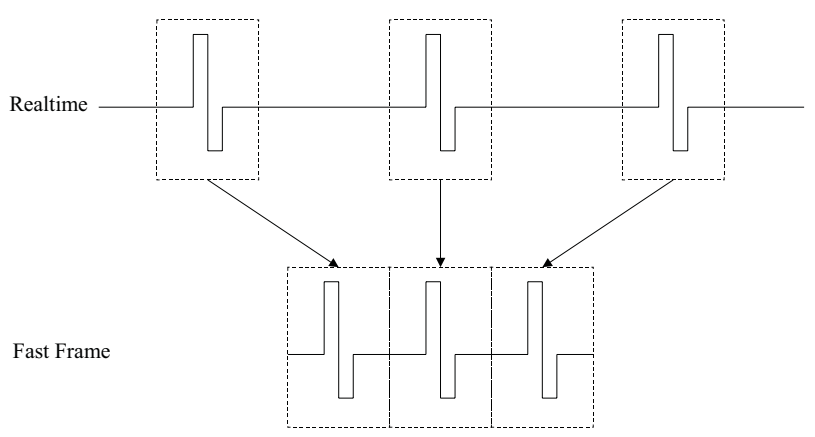

Fig. 2. Principle of fast frame mode.

\subsection{Trigger time measurement}

The used oscilloscope needs to offer a fast frame mode. In this mode the oscilloscope captures the absolute times of trigger events which occur in sequence. In case the oscilloscope triggers on pulses, the times between the pulses can calculated. The principle of the fast frame mode is shown in Fig. 2. A measurement of a certain amount of trigger times, a calculation of the reliable statistics about which times between pulses occur mostly. Figure 3 shows an example for a probability density estimate calculated over times between pulses radiated by a hand-held drill machine. The times which correlate to the local maxima of the graph will be used for signal reconstruction. They are randomly but according to their relative frequency of occurrence distributed on the timescale. An example for a reconstructed timescale is shown in Fig. 4.

\subsection{Signal measurement}

The sampling rate $f_{s}$ must meet the Nyquist requirements. Recording occurs in a manner of several single shot measurements. Time per division on the oscilloscope must be set that single pulses fit in single records. The recorded pulse is used for signal reconstruction only if it meets the following requirements: Firstly the pulse does not clip and secondly it is unique which means, the pulse is in a spectral comparison to already saved pulses not correlated or similar to one of those. This leads to a enormous data reduction. From the record of a high amount of pulses, a reliable estimation is calculated. The estimation gives an information in what relation the frequency of occurrence of the pulses stand to each other. Weighed with that estimation, the pulses are randomly

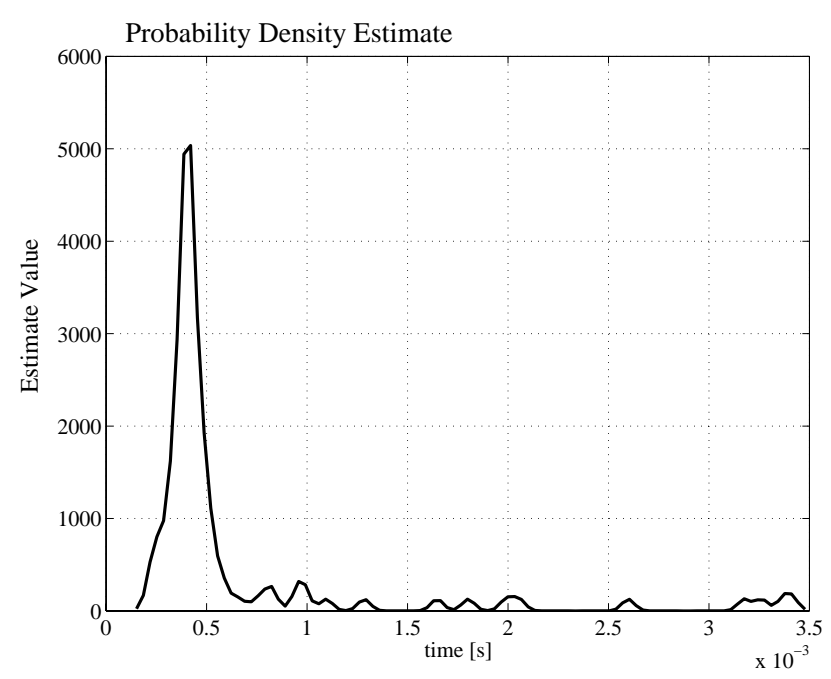

Fig. 3. Probability density estimation for the emission of a drill machine.

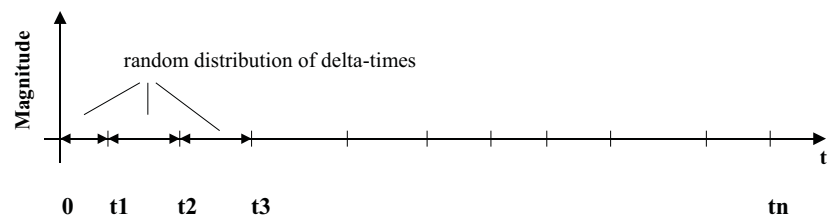

Fig. 4. Reconstructed timescale from the probability density estimation.

distribute on the marks of the timescale as shown in Fig. 4. The result of the pulses distribution on the reconstructed timescale is shown in Fig. 5. As the signal contains pulses of varying amplitudes, the routine which is described above needs to be done for different oscilloscope vertical resolutions. The oscilloscope trigger-level must be adapted to each vertical oscilloscope setting (e.g. fix trigger level on the oscilloscope screen). This ensures improvement of the measurement dynamic range. From this results as many timescales as many vertical scales have measured. Last step is to merge these timescales together to one signal representation.

\subsection{Merger to one signal representation}

That procedure of merge to one signal representation is shown for the example of two timescales in Fig. 6. In the fast frame mode the oscilloscope also triggers on pulses which clip. That means the times between trigger events measured consider the clipping pulses. So randomly distributed pulses on the timescale of the measurement with higher resolution are replaced by the randomly distributed pulses of the measurement at lower oscilloscope resolution. This needs to be done at places where they overlap. In case there exist measurements for further oscilloscope vertical settings, the procedure described above would be done iteratively. 


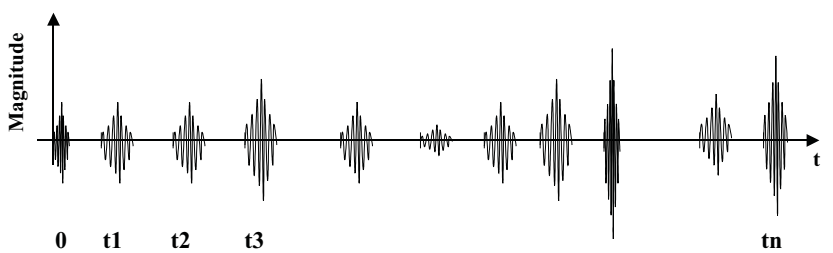

Fig. 5. Pulses distribution on the reconstructed timescale.

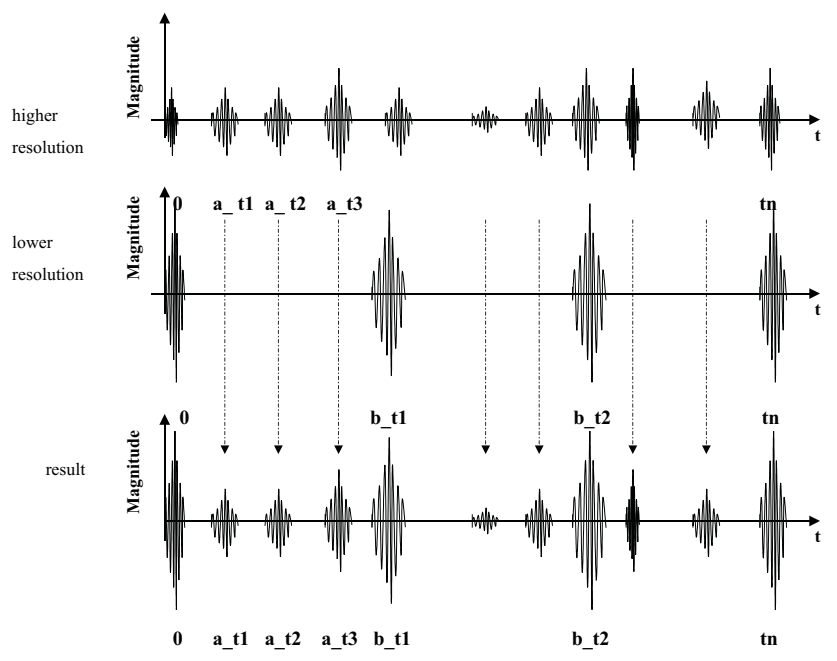

Fig. 6. Example of a signal reconstruction.

\section{Automated TDEMI measurement algorithm for class A signals}

In case the signal shows no pulsar envelope, or if times between pulses are shorter than fast frame mode is able to follow updating trigger times (e.g. limit of oscilloscope Tektronix TDS 7154 is $6 \mu \mathrm{s}$ ), doing statistical analysis brings no merit. A detailed measurement description of this class of signal have already been presented in Krug (2002c).

\section{Spectral estimation}

\subsection{Windowing}

Spectral estimation is done with use of Discrete Fourier Transform (DFT). DFT computes the amplitude spectrum on a time record of length $N$. In addition DFT is based upon the assumption that this time record is repeated throughout time. So the time record $x[n]$ needs to be multiplied with a window $w[n]$ of length $N$ to avoid spectral leakage:

$z[n]=x[n] w[n]$.

The window function is equivalent to the bandwidth of the Intermediate-Frequency-filter (IF-filter) of the conventional EMI-Receiver. The window function needs to be adapted to the IF-filter regarding impulse-bandwidth and equivalent noise-bandwidth. Appropriately the IF-filter is modelled

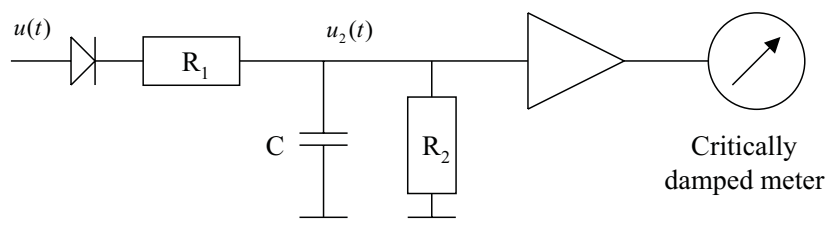

Fig. 7. Analog Quasi-Peak-Detektor.

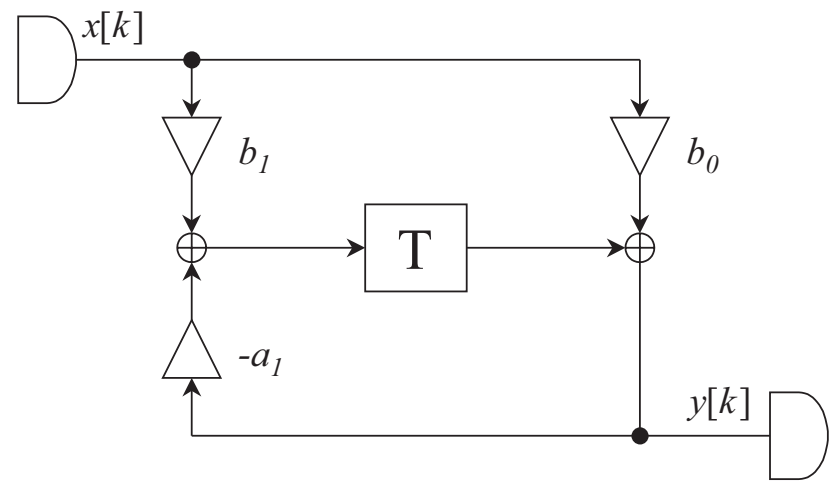

Fig. 8. Signal flow of a IIR1-filter.

with a gaussian window. The DFT being applied to each of the data blocks and is defined as follows (Marple, 1987):

$Z[k]=\sum_{n=0}^{N-1} z[n] e^{-j \frac{2 \pi k n}{N}} \quad n=1 \ldots N, k=1 \ldots N$

\subsection{Short-time-Fourier-transform}

In order to receive the filter response in form of a time dependent amplitude spectrum, a DFT calculation using record samples less than the length of the time record $N$ is necessary. The process of iteratively shifting the window along the time record followed by computing a DFT is the Short-TimeDiscrete-Fourier-Transform (STFT) (Cohen, 1989). The window shift is done with an appropriate increment. The time domain resolution $T_{S B B}$ of the calculated amplitude spectra is important for an accurate filter response. $T_{S B B}$ corresponds with the increment used in the STFT as follows (Cohen, 1989):

$T_{S B B}=\frac{1}{f_{S B B}}$

$f_{S B B}$ is the baseband sampling frequency. The signal representation in Fig. 6 and time-response for each pulse calculated via STFT is given to the model of the Average-, Peak-, RMS,- and Quasi-Peak-Detector.

\section{Detector model}

\subsection{Analog Quasi-Peak-Detector}

In Fig. 7 the circuit of a analog Quasi-Peak-Detector is shown. The demodulated signal $u(t)$ of the conventional 
Table 1. CISPR specifications for Quasi-Peak-Detector.

\begin{tabular}{cccc}
\hline \multicolumn{3}{c}{ Frequency range } \\
\hline & Band A & Band B & Band C/D \\
& $9-150 \mathrm{kHz}$ & $0,15-30 \mathrm{MHz}$ & $30-1000 \mathrm{MHz}$ \\
\hline IF 6dB Bandwidth & $0,2 \mathrm{kHz}$ & $9 \mathrm{kHz}$ & $120 \mathrm{kHz}$ \\
$\tau_{c}$ & $45 \mathrm{~ms}$ & $1 \mathrm{~ms}$ & $1 \mathrm{~ms}$ \\
$\tau_{d}$ & $500 \mathrm{~ms}$ & $160 \mathrm{~ms}$ & $550 \mathrm{~ms}$ \\
$\tau_{m}$ & $160 \mathrm{~ms}$ & $160 \mathrm{~ms}$ & $100 \mathrm{~ms}$ \\
\hline
\end{tabular}

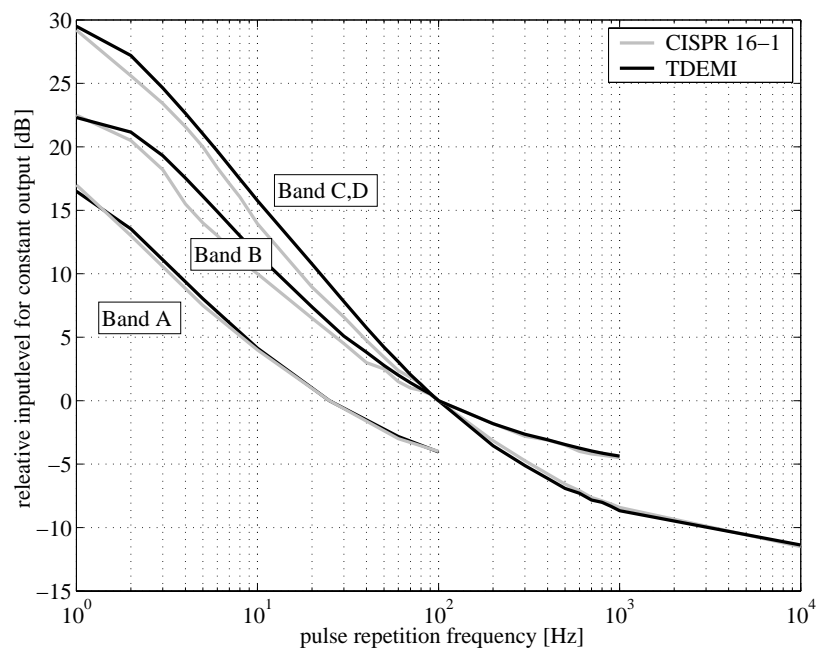

Fig. 9. CISPR 16-1 and TDEMI-System pulse response curve.

EMI-Receiver charges the capacitor $C$ by the resistor $R_{1}$ as long as $u(t)$ is above $u_{2}(t)$. This is a typical RC-charging with the time constant $\tau_{c}$. If the the input signal $u(t)$ is lower than $u_{2}(t)$, the voltage $u_{2}(t)$ is discharged by resistor $R_{2}$. A critically damped meter with the time constant $\tau_{m}$ is used to display the amplitude. A buffer is necessary to avoid any repercussions. The maximum of the shown value is taken as Quasi-Peak-Value. The values of time constants and IF bandwidths for CISPR 16-1 (CISPR16-1, 1999) are shown in Table 1.

For proper Quasi-Peak-Detection the input signal must be provided up to $2 \mathrm{~s}$, to have a ready steady state. The problem of todays Quasi-Peak-Detectors is that provide the Quasi-Peak-value of a single frequency within $2 \mathrm{~s}$. For about 15000 frequency points, in a normal CISPR C, D measurement about $9 \mathrm{~h}$ are necessary.

\subsection{Digital Quasi-Peak-Detector model}

For the integration of a Quasi-Peak-Detector into a TDEMISystem it is necessary to provide a digital equivalent realization, that can be run at least 10 times faster than the analog equivalent. $u(t)$ is provided by intelligent recording technique and a spectral estimation via STFT. For modelling the charging and discharging process digital IIR1-filters are

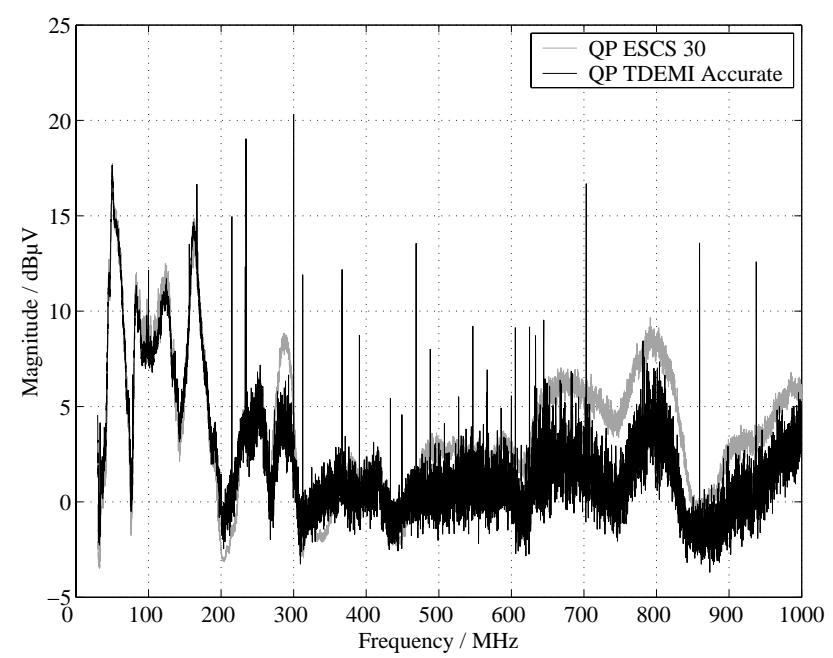

Fig. 10. Drill machine: Comparison between TDEMI-System and conv. EMI-Receiver.

used. Figure 8 shows the signal flow of a IIR1-filter. For calculating the filter coefficients following transfer function is used:

$H(s)=\frac{1}{1+\tau s}$.

Also the bilinear transformation for characterizing the RCsystem is used. The bilinear transformation is defined as follows:

$s=\frac{2}{\triangle t} \frac{z-1}{z+1}$.

$s$ is the frequency-domain variable, $z$ is the discrete timedomain variable and $\Delta t$ is the time-domain discretisation. In order to get better numeric precision not the normal topology of a IIR2-system, but a cascading of two identical IIR1-filters for modelling the critically damped meter were used. The maximum of the output signal is taken as Quasi-Peak-Value. Charging and discharging is decided by a comparator, while in discharging mode the coefficient $b_{0}$ is set to zero. The model of the digital Quasi-Peak-Detector was written for a conventional Intel Pentium III $1 \mathrm{GHz}$ processor, with the use of intrinsics. Therefore simulation time of a single QuasiPeak-value is about $0.04 \mathrm{~s}$.

\subsection{Signal representation for Quasi-Peak-Detection}

A main problem is providing a suitable signal for the QuasiPeak-Detector. By recording up to $2 \mathrm{~s}$ with a TDEMI-System at $5 \mathrm{GS} / \mathrm{s}$ at least $10 \mathrm{Gbyte}$ RAM necessary. Therefore the signal is reconstructed from representative time-domain signal parts and their corresponding time stamps. The reconstruction is done after the STFT and the demodulation. A time-domain signal is created for $2 \mathrm{~s}$ and is estimated by the Quasi-Peak-Detector. The sampling frequency of the timedomain signal must be chosen properly to minimize the error between the digital and the analog implementation. For 


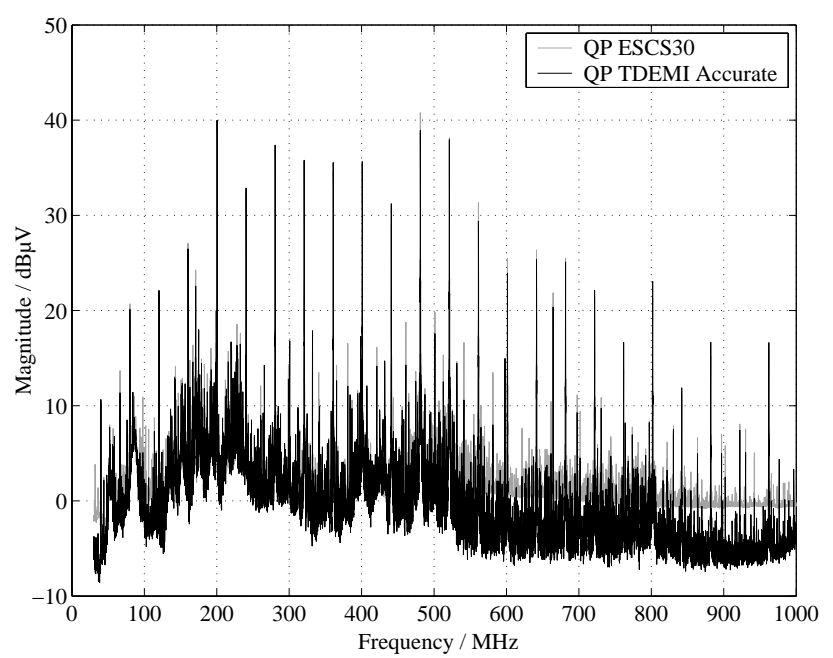

Fig. 11. Laptop: Comparison between TDEMI-System and conv. EMI-Receiver.

Band CD (CISPR16-1, 1999) about $500 \mathrm{kHz}$ is sufficient. The reconstruction is equivalent to reconstructing the signal in the time-domain and applying the STFT and Quasi-PeakDetection, as long as the pulse response of the IF-filter do not overlap. The error is minimized by selecting the appropriate mode of the intelligent recording algorithm.

\section{Measurement results}

\subsection{Simulation CISPR 16-1 pulse}

For approving the Quasi-Peak-Detection in time-domain the CISPR 16-1 pulse response curves simulated at a single frequency is shown in Fig. 9. The CISPR 16-1 and the TDEMISystem pulse response curve show a math for all pulse repetition frequencies.

\subsection{Emission measurement}

Figure 10 shows the result of a measurement with the TDEMI-System on a drill-machine in band C,D for QuasiPeak-Detection. The average deviation over the whole frequency range is below $3 \mathrm{~dB}$. The drill-machine emit pulses with irregular repetition frequency. Pulses of the drillmachine extremely differ in amplitude and spectrum. The automated TDEMI measurement algorithm for class $\mathrm{C}$ signals was used. Figure 11 shows the result of a measurement with the TDEMI-System on a Pentium $200 \mathrm{MHz}$ Laptop in band $\mathrm{C}, \mathrm{D}$. The average deviation over the whole frequency range is below $3 \mathrm{~dB}$. A laptop emit pulses with a high, not regular repetition frequency. The automated TDEMI measurement algorithm for class A signals was used. In Fig. 12 a measurement with the TDEMI-System of a SVGA-Monitor in band $\mathrm{C}$ is shown. A good match of the emission measured with the conventional EMI-Receiver and the TDEMI-System is shown. Even the low spectral lines of the monitor power

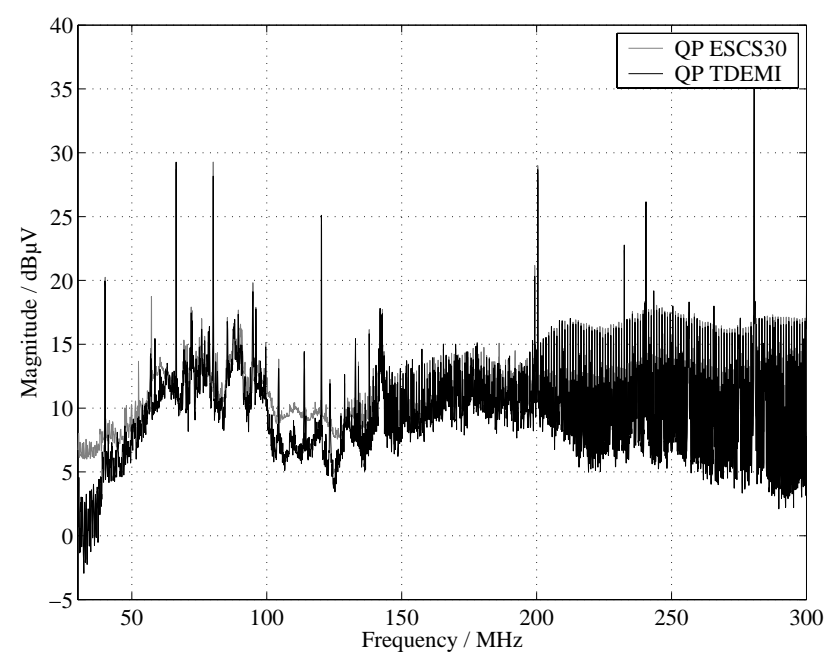

Fig. 12. SVGA Monitor: Comparison between TDEMI-System and conv. EMI-Receiver.

supply show a maximum difference below $1.5 \mathrm{~dB}$. The monitor emit pulses with a high but constant repetition frequency. The automated TDEMI measurement algorithm for class A signals was used.

\section{Conclusion}

The presented broad-band time-domain electromagnetic interference measurement system allows to emulate in realtime the various modes of conventional detector modes. With the presented time domain measurement system the measurement time can be reduced by a factor of 10 . A novel signal recording routine for time-domain EMI (TDEMI) measurements and Quasi-Peak-Detection is described. Measurement results obtained from the investigation of a drill machine, monitor and laptop obtained with the time-domain electromagnetic interference (TDEMI) measurement system are discussed. The results obtained with the described system have been compared with measurements performed with a conventional EMI receiver.

\section{References}

Krug, F. and Russer, P.: Time-domain Broad-band EMI Measurement Techniques, 32th European Microwave Conference, Milan, Italy, 23-27 September 2002, 641-644, 2002a.

Krug, F. and Russer, P.: Ultra-fast broadband EMI measurement in time domain using classical spectral estimation, 2002 IEEE MTT-S International Microwave Symposium Digest, 2-6 June, Seattle, USA, 2237-2240, 2002b.

Krug, F. and Russer, P.: Ultra-fast broadband EMI time-domain measurement system, 2002 International Symposium On Electromagnetic Compatibility Digest, 9-13 September, Sorrento, Italy, 379-384, 2002c. 
Krug, F. and Russer, P.: The Time-Domain Electromagnetic Interference Measurement System, IEEE Transactions on Electromagnetic Compatibility, 45, 2, 330-338, 2003.

Marple, S.-L.: Digital Spectral Analysis with Applications, ISBN 08493-7892-3, Prentice-Hall, 1987.
Cohen, L.: Time-Frequency Distributions - A Review, Proceeding of the IEEE, 77, 7, 941-981, 1989.

CISPR16-1: Specification for radio disturbance and immunity measuring apparatus and methods Part 1: Radio disturbance and immunity measuring apparatus, International Electrotechnical Commission, 1999. 